\title{
Psychological perspectives on expertise
}

\author{
Guillermo Campitelli ${ }^{1 *}$, Michael H. Connors ${ }^{2,3}$, Merim Bilalić ${ }^{4}$ and David Z. Hambrick ${ }^{5}$ \\ 1 School of Psychology and Social Science, Edith Cowan University, Joondalup, WA, Australia, ${ }^{2}$ Department of Cognitive \\ Science, ARC Centre of Excellence in Cognition and its Disorders, Macquarie University, Sydney, NSW, Australia, ${ }^{3}$ Dementia \\ Collaborative Research Centre, School of Psychiatry, University of New South Wales, Sydney, NSW, Australia, ${ }^{4}$ Department \\ of General Psychology and Cognitive Science, Institute of Psychology, Alpen Adria University Klagenfurt, Klagenfurt, Austria, \\ ${ }^{5}$ Department of Psychology, Michigan State University, East Lansing, MI, USA
}

Keywords: expertise, expert performance, expert cognitive processes, skill acquisition, skill transfer, training, deliberate practice

\section{Introduction}

This Research Topic sought to advance psychological understanding of expertise by drawing together lines of research from many different domains of expertise. The outcome is a collection of 35 articles in such diverse areas as chess, music, perception, teaching, intensive-care diagnosis, video-games, sports, dance, mathematics, climbing, and fingerprint analysis.

The articles can be classed into five broad categories based on their focus: (a) the cognitive processes in expertise, (b) the development of expertise, (c) the relationship between expertise and general cognition, (d) the transfer of skills between domains, and (e) methodological issues and frameworks in expertise research. We give a brief overview of the research across these five themes.

\section{Cognitive Processes in Expertise}

Articles in this research topic used a number of different methodologies to investigate cognitive processes in expertise. Four articles examined experts' eye movements as a way of studying what experts focus on when performing domain-relevant tasks. Sheridan and Reingold (2014), for example, found that expert chess players rapidly differentiate regions of the board that are relevant to the best move from irrelevant ones. Similarly, McCormack et al. (2014) found that expert intensive-care physicians directed their attention to more relevant areas of the situation, compared to competent non-experts. In addition, Godau et al. (2014) found that experts in arithmetic problem solving spontaneously used arithmetic shortcuts. Finally, Ellis and Reingold (2014) examined the Einstellung effect (i.e., where the first idea that comes to mind blocks finding the best solution to a problem) using this methodology and noted its relevance to understanding expert flexibility (see Bilalić and McLeod, 2014).

Two articles focused on perceptual expertise. Curby and Gauthier (2014) found that acquiring expertise with a category of stimuli (i.e., car expertise) increases the interference between the visual processing of other familiar stimuli (e.g., faces) and that of the learned category (cars). In a study with novel objects, Cheung and Gauthier (2014) found that acquiring perceptual expertise involves integrating perceptual and conceptual representations of stimuli.

Four studies investigated expertise involving physical movements. In a study on dancing, Bläsing (2015) found that making a sequence of movements influenced the subsequent perception of that sequence, but not to the same degree if one was a dancing expert. In a study of climbing experts, Bläsing et al. (2014) found that expertise was associated with better perception of climbing holds and action-relevant objects. In a study of athletes and musicians, Braun Janzen et al. (2014) showed that training affects performance involving timing and rhythmic 
movements: athletes were more precise making continuous movements, whereas musicians were more precise for discrete movements. In a study of skill acquisition in a flight simulator, Wiggins et al. (2014) found that a general capacity for acquiring and using cues was related to performance in landing an aircraft in the simulator.

Finally, four articles focused on experts' pattern recognition (the ability to identify meaningful relationships in complex stimuli). In a review of research on fingerprint experts, Thompson et al. (2014) concluded that such expertise relies on rapid pattern recognition and discrimination rather than in analytic thinking. In an observational study, Kretz and Krawczyk (2014) found that academic economists use many analogies in research meetings. Trench (2014), however, suggests that these results may be due to the naturalistic setting of the study, rather than expertise per se. Bialek and Sawicki (2014) showed that participants asked to take an expert perspective become more risk aversive and patient in decision making tasks. Finally, Leone et al. (2014) examined the relationship between expertise and representations of space using a large dataset of chess games from an internet server. They found that novices, relative to experts, use strategies to reduce their cognitive load (see Connors and Campitelli, 2014, for a commentary).

\section{Development of Expertise}

Six studies examined how expertise is developed. Gaschler et al. (2014) examined the learning curves in skill acquisition by analyzing the tournament performance of 1383 chess players over 10 years. They found that exponential learning curves better fitted players' improvements over time than power function learning curves. Gobet and Ereku (2014) discussed the case of Magnus Carlsen, current world chess champion, and argue that his level of performance cannot be accounted for by the deliberate practice account, which suggests that amount of deliberate practice is the critical determinant of expertise.

Citing limitations in an earlier meta-analysis by Hambrick et al. (2014a), Platz et al. (2014) conducted a meta-analysis on the influence of deliberate practice in musical achievement. They found a moderate average effect size $\left(r_{c}=0.61\right)$, which they interpret as showing the importance of deliberate practice. In response to Platz et al.'s criticisms, Hambrick et al. (2014b) noted a number of conceptual problems in Platz et al.'s arguments and observe how Platz et al.'s findings can also be interpreted to show that practice, while undoubtedly an important factor in expertise, is not the sole determinant.

Healy et al. (2014) proposed a number of training principles for developing expertise. These include the acquisition of expertise (e.g., scheduling of feedback), retention of expertise (e.g., item chunking, depth of processing) and transfer (e.g., variability of practice, seeding the knowledge base). Finally, Speelman (2014) argued that treating numeracy as a form of expertise and using computer programs in teaching would address some shortcomings in current teaching and, in particular, foster a greater focus on practice and feedback in learning.

\section{Expertise and General Cognition}

Three articles examined the relationship between expertise and general cognition. First, Gobet et al. (2014b) discussed how artificial intelligence and engineering could be used to design a brain. Based on expertise research, they propose that a better brain would have less concepts and more low-level perceptual processing. Second, Guida and Lavielle-Guida (2014) combined findings from memory research with the normal population with theories of expert memory. They argue that a less sophisticated version of the spatial method of loci used by memory experts is also used by ordinary people to encode items in working memory. Third, Christophel et al. (2014) observed that amount of teaching experience is a very poor predictor of a teacher's actual effectiveness, including, for example, the teacher's ability to offer constructive feedback to students.

\section{Transfer of Skills}

Three articles examined the transfer of skills across domains. First, Gobet et al. (2014a) investigated the possibility of training transfer from videogame playing to selective attention and working memory capacity. Consistent with over a century of research, there was no evidence for transfer, even in videogame experts. In contrast, Lampit et al. (2014) reported evidence for transfer of computerized cognitive training to a bookkeeping task. Finally, Bart (2014) reviewed research published after Gobet and Campitelli's (2006) critical review on the effects of chess education, showing statistically significant effects of chess education on academic achievement.

\section{Frameworks, Recommendations, and Methodology}

Seven articles discussed expertise in general. First, Vaci et al. (2014) consider alternative approaches to studying expertise, and in particular, how studying only individuals from highly restricted ranges of skill may yield different findings than studying individuals who represent wider ranges of skill. Second, Kaufman (2014) identifies points of disagreement and agreement in different views of expertise and suggests some directions for future research. Third, Bourne et al. (2014), categorize expertise as elite, peak, or exceptionally high levels of performance on a particular task or within a given domain.

Fourth, Shen et al. (2014) use birdwatching as an illustrative example to discuss such issues as selecting an appropriate domain of perceptual expertise for study, recruiting experts, assessing their level of expertise, and experimentally testing the experts' performance. Fifth, MacIntyre et al. (2014) propose that athletes are not just experts in movement execution but also in planning, metacognition, and reflection. Similarly, Toner and Moran (2014), extending Sutton et al.'s 
(2011) framework, argue that expert athletes do not completely automatize their skills and that an important component of their expertise is to be able to rapidly reflect on their movements. Finally, de Oliveira et al. (2014) build upon Gigerenzer's (e.g., Gigerenzer and Goldstein, 1999) heuristicbased approach to decision making. They propose that expert athletes develop a toolbox of heuristics to guide their decision making.

\section{References}

Bart, W. M. (2014). On the effect of chess training on scholastic achievement. Front. Psychol. 5:762. doi: 10.3389/fpsyg.2014.00762

Bialek, M., and Sawicki, P. (2014). Can taking the perspective of an expert debias human decisions? The case of risky and delayed gains. Front. Psychol. 5:989. doi: 10.3389/fpsyg.2014.00989

Bilalić, M., and McLeod, P. (2014). Why good thoughts block better ones. Sci. Am. 310, 74-79. doi: 10.1038/scientificamerican0314-74

Bläsing, B. E. (2015). Segmentation of dance movement: effects of expertise, visual familiarity, motor experience and music. Front. Psychol. 5:1500. doi: 10.3389/fpsyg.2014.01500

Bläsing, B. E., Güldenpenning, I., Koester, D., and Schack, T. (2014). Expertise affects representation structure and categorical activation of grasp postures in climbing. Front. Psychol. 5:1008. doi: 10.3389/fpsyg.2014.01008

Bourne, L. E. Jr., Kole, J. A., and Healy, A. F. (2014). Expertise: defined, described, explained. Front. Psychol. 5:186. doi: 10.3389/fpsyg.2014.00186

Braun Janzen, T., Thompson, W. F., Ammirante, P., and Ranvaud, R. (2014). Timing skills and expertise: discrete and continuous timed movements among musicians and athletes. Front. Psychol. 5:1482. doi: 10.3389/fpsyg.2014. 01482

Cheung, O. S., and Gauthier, I. (2014). Visual appearance interacts with conceptual knowledge in object recognition. Front. Psychol. 5:793. doi: 10.3389/fpsyg.2014.00793

Christophel, E., Gaschler, R., and Schnotz, W. (2014). Teachers' expertise in feedback application adapted to the phases of the learning process. Front. Psychol. 5:858. doi: $10.3389 /$ fpsyg. 2014.00858

Connors, M. H., and Campitelli, G. (2014). Expertise and the representation of space. Front. Psychol. 5:270. doi: 10.3389/fpsyg.2014.00270

Curby, K. M., and Gauthier, I. (2014). Interference between face and non-face domains of perceptual expertise: a replication and extension. Front. Psychol. 5:955. doi: 10.3389/fpsyg.2014.00955

de Oliveira, R. F., Lobinger, B. H., and Raab, M. (2014). An adaptive toolbox approach to the route to expertise in sport. Front. Psychol. 5:709. doi: 10.3389/fpsyg.2014.00709

Ellis, J. J., and Reingold, E. M. (2014). The Einstellung effect in anagram problem solving: evidence from eye movements. Front. Psychol. 5:679. doi: 10.3389/fpsyg.2014.00679

Gaschler, R., Progscha, J., Smallbone, K., Ram, N., and Bilalić, M. (2014). Playing off the curve - testing quantitative predictions of skill acquisition theories in development of chess performance. Front. Psychol. 5:923. doi: 10.3389/fpsyg.2014.00923

Gigerenzer, G., and Goldstein, D. G. (1999). "Betting on one good reason: the take the best heuristic," in Simple Heuristics that Make us Smart, eds G. Gigerenzer, P. M. Todd, and The ABC Research Group (New York, NY: Oxford University), 75-95.

Gobet, F., and Campitelli, G. (2006). "Education and chess: a critical review," in Chess and Education: Selected Essays from the Koltanowski Conference, ed T. Redman (Dallas, TX: Chess Program at the University of Texas at Dallas), 124-143.

Gobet, F., and Ereku, M. H. (2014). Checkmate to deliberate practice: the case of Magnus Carlsen. Front. Psychol. 5:878. doi: 10.3389/fpsyg.2014.00878

Gobet, F., Johnston, S. J., Ferrufino, G., Johnston, M., Jones, M. B., Molyneux, A., et al. (2014a). "No level up!": no effects of video game specialization

\section{Conclusion}

The diversity of articles in this research topic illustrates the many different approaches to studying expertise. It also indicates the keen interest in the topic. We believe that many articles in this research topic are of lasting importance and can help to guide future research in the field of expertise.

and expertise on cognitive performance. Front. Psychol. 5:1337. doi: 10.3389/fpsyg.2014.01337

Gobet, F., Snyder, A., Bossomaier, T., and Harré, M. (2014b). Designing a "better" brain: insights from experts and savants. Front. Psychol. 5:470. doi: $10.3389 /$ fpsyg.2014.00470

Godau, C., Haider, H., Hansen, S., Schubert, T., Frensch, P. A., and Gaschler, R. (2014). Spontaneously spotting and applying shortcuts in arithmetica primary school perspective on expertise. Front. Psychol. 5:556. doi: 10.3389/fpsyg.2014.00556

Guida, A., and Lavielle-Guida, M. (2014). 2011 space odyssey: spatialization as a mechanism to code order allows a close encounter between memory expertise and classic immediate memory studies. Front. Psychol. 5:573. doi: 10.3389/fpsyg.2014.00573

Hambrick, D. Z., Altmann, E. M., Oswald, F. L., Meinz, E. J., and Gobet, F. (2014b). Facing facts about deliberate practice. Front. Psychol. 5:751. doi: 10.3389/fpsyg.2014.00751

Hambrick, D. Z., Oswald, F. L., Altmann, E. M., Meinz, E. J., Gobet, F., and Campitelli, G. (2014a). Deliberate practice: is that all it takes to become an expert? Intelligence 45, 34-45. doi: 10.1016/j.intell.2013.04.001

Healy, A. F., Kole, J. A., and Bourne, L. E. Jr. (2014). Training principles to advance expertise. Front. Psychol. 5:131. doi: 10.3389/fpsyg.2014.00131

Kaufman, S. B. (2014). A proposed integration of the expert performance and individual differences approaches to the study of elite performance. Front. Psychol. 5:707. doi: 10.3389/fpsyg.2014.00707

Kretz, D. R., and Krawczyk, D. C. (2014). Expert analogy use in a naturalistic setting. Front. Psychol. 5:1333. doi: 10.3389/fpsyg.2014.01333

Lampit, A., Ebster, C., and Valenzuela, M. (2014). Multi-domain computerized cognitive training program improves performance of bookkeeping tasks: a matched-sampling active-controlled trial. Front. Psychol. 5:794. doi: 10.3389/fpsyg.2014.00794

Leone, M. J., Fernandez Slezak, D., Cecchi, G. A., and Sigman, M. (2014). The geometry of expertise. Front. Psychol. 5:47. doi: 10.3389/fpsyg.2014.00047

MacIntyre, T. E., Igou, E. R., Campbell, M. J., Moran, A. P., and Matthews, J. (2014). Metacognition and action: a new pathway to understanding social and cognitive aspects of expertise in sport. Front. Psychol. 5:1155. doi: 10.3389/fpsyg.2014.01155

McCormack, C., Wiggins, M. W., Loveday, T., and Festa, M. (2014). Expert and competent non-expert visual cues during simulated diagnosis in intensive care. Front. Psychol. 5:949. doi: 10.3389/fpsyg.2014.00949

Platz, F., Kopiez, R., Lehmann, A. C., and Wolf, A. (2014). The influence of deliberate practice on musical achievement: a meta-analysis. Front. Psychol. 5:646. doi: 10.3389/fpsyg.2014.00646

Shen, J., Mack, M. L., and Palmeri, T. J. (2014). Studying real-world perceptual expertise. Front. Psychol. 5:857. doi: 10.3389/fpsyg.2014.00857

Sheridan, H., and Reingold, E. M. (2014). Expert vs. novice differences in the detection of relevant information during a chess game: evidence from eye movements. Front. Psychol. 5:941. doi: 10.3389/fpsyg.2014.00941

Speelman, C. P. (2014). The acquisition of expertise in the classroom: are current models of education appropriate? Front. Psychol. 5:580. doi: 10.3389/fpsyg.2014.00580

Sutton, J., Mcilwain, D., Christensen, W., and Geeves, A. (2011). Applying intelligence to the reflexes: embodied skills and habits between dreyfus and descartes. J. Br. Soc. Phenomenol. 42, 78-103. doi: 10.1080/00071773.2011. 11006732 
Thompson, M. B., Tangen, J. M., and Searston, R. A. (2014). Understanding expertise and non-analytic cognition in fingerprint discriminations made by humans. Front. Psychol. 5:737. doi: 10.3389/fpsyg.2014.00737

Toner, J., and Moran, A. (2014). In praise of conscious awareness: a new framework for the investigation of "continuous improvement" in expert athletes. Front. Psychol. 5:769. doi: 10.3389/fpsyg.2014.00769

Trench, M. (2014). Explaining the abundance of distant analogies in naturalistic observations of experts. Front. Psychol. 5:1487. doi: 10.3389/fpsyg.2014. 01487

Vaci, N., Gula, B., and Bilalić, M. (2014). Restricting range restricts conclusions. Front. Psychol. 5:569. doi: 10.3389/fpsyg.2014.00569

Wiggins, M. W., Brouwers, S., Davies, J., and Loveday, T. (2014). Traitbased cue utilization and initial skill acquisition: implications for models of the progression to expertise. Front. Psychol. 5:541. doi: 10.3389/fpsyg. 2014.00541

Conflict of Interest Statement: The authors declare that the research was conducted in the absence of any commercial or financial relationships that could be construed as a potential conflict of interest.

Copyright $\odot 2015$ Campitelli, Connors, Bilalić and Hambrick. This is an open-access article distributed under the terms of the Creative Commons Attribution License (CC $B Y$ ). The use, distribution or reproduction in other forums is permitted, provided the original author(s) or licensor are credited and that the original publication in this journal is cited, in accordance with accepted academic practice. No use, distribution or reproduction is permitted which does not comply with these terms. 